\title{
Brain arrest to neurological determination of death to organ utilization: the evolution of hospital- based organ donation strategies in Canada
}

Sam D. Shemie MD

$\mathrm{H}$ ISTORICALLY, organ donation strategies have grown out of efforts by the transplant communities to meet the needs of their potential transplant recipients. While successful to some degree, cadaveric organ donation rates in Canada have not improved. The increasing gap between transplant needs and organ availability is well known and has stimulated both provincial and federal government action.

In September 1999, the Federal, Provincial and Territorial (FPT) Ministers of Health agreed to formulate a coordinated FPT strategy to improve the organ and tissue donation and transplantation system in Canada. In April 2001, a key component of this strategy was the announcement to establish the Canadian Council for Donation and Transplantation (CCDT; www.ccdt.ca). The mandate of the Council is to provide advice to the FPT Conference of Deputy Ministers of Health in support of their efforts to coordinate FPT activities relating to organ and tissue donation and transplantation.

The brain dead organ donor is the principle source of transplantable organs in Canada. Paradoxically, the care of these individuals is the exclusive domain of the intensive care unit (ICU). As transplant specialties have no role in the care of potential organ donors, their ability to influence this area of practice is limited. The Donation Committee of the CCDT recognized the necessity of engaging and supporting the ICU community to assume leadership in establishing standards for optimal practice in this field. Concurrently, the Canadian Critical Care Society (CCCS) established a working group on organ and tissue donation, ${ }^{1}$ expressing strong support for collaborative initiatives to develop, implement and evaluate processes to increase organ and tissue donation within sound ethical and legal frameworks. The CCCS emphasized that the option of organ and tissue donation should be offered to all eligible families and be considered a standard component of quality end-of-life care in the ICU.

As there are misconceptions often associated with the term brain death, it is better understood as brain arrest. It is analogous to the event of a cardiac arrest - once irreversible, the individual's death is subsequently determined by cardiocirculatory criteria based on the absence of heartbeat and circulation. Once brain arrest occurs and is irreversible, death is subsequently determined by neurological criteria based on the absence of brainstem function. This neurological determination of death is the process and procedure to determine death.

In this, and recent issues of the Canadian Journal of Anesthesia, a series of manuscripts appear which support recently developed consensus recommendations for the neurological determination of death ${ }^{2}$ and organ donor management. ${ }^{3}$ These manuscripts are the result of national collaborative efforts to improve the process and procedures to actualize the desire and opportunity for Canadians to donate. They have practice implications for staff in the emergency department, neurosciences, critical care, anesthesia, surgery and the operating room.

The pathway to organ donation begins with a severe and devastating form of brain injury that deteriorates to brain arrest. If the arrest of the brain is complete and irreversible, diagnostic procedures should be applied to confirm the neurological determination of death. Families should be offered the option of donation and if consent is provided, this culminates in surgical procurement of organs from consented donors. The first CCDT initiative, "From Severe Brain Injury

From the Division of Pediatric Critical Care, Montreal Children's Hospital, McGill University Health Centre, Montreal, Quebec; and the Canadian Critical Care Society, Canadian Council for Donation and Transplantation, Canada.

Address correspondence to: Dr. Sam D. Shemie, Division of Pediatric Critical Care, Montreal Children's Hospital, McGill University Health Centre, 2300 Tupper Street, Montreal, Quebec H3H 1P3, Canada. Phone: 514-412-4400, ext. 23806; Fax: 514-412-4205; E-mail: sam.shemie@muhc.mcgill.ca 
to Neurological Determination of Death" held April 9-11 $1^{\text {th }} 2003$ in Vancouver, discussed the path and processes influencing this interval of care. ${ }^{4}$

Once the brain is severely injured, the patient must reach the emergency department (ED) and be transferred to the ICU for neuroprotective therapies or end-of-life care. Characteristics of ED donor recognition and ED to ICU admission triage in Ontario neurosurgical centres (ONCs) is reviewed by Tenn-Lyn et al. ${ }^{5}$ A significant number of patients transferred to ONCs have an injury with a likelihood of progressing to brain death, but only a small proportion of these patients are recognized as such. Rocker et al. ${ }^{6}$ discuss practice variation in end-of-life care in the ICU that may have significant implications for patients with severe brain injury. Evolving neuroprotective therapies may improve outcomes in severe brain injury ${ }^{7-9}$ and thus may challenge the way treatment, juxtaposed to end-of-life decisions, are being made in the ED and ICU.

Historical perspectives and the evolution of brain death criteria are provided by Baron et al. ${ }^{10}$ Once complete and irreversible brain arrest occurs, it must be recognized and reliably diagnosed with clarity and consistency. Despite being sound in concept, variabilities in recommendations and practice are widespread. Guidelines established by the Canadian Congress Committee on Brain Death ${ }^{11}$ in 1988 and the Canadian Neurocritical Care Group ${ }^{12}$ in 1999 initiated clarification of criteria, but have not lead to uniform practice. Hornby et al. ${ }^{13}$ review Canadian hospital based adult and pediatric brain death guidelines, confirming the substantial and concerning inconsistencies in practices $^{14,15}$ and documentation ${ }^{16}$ described in other countries. These observations confirm the need for a national standard to address diagnostic variability and improve documentation, as corroborated by a survey of Canadian critical care physicians by Doig et al. ${ }^{17}$ While the neurological determination of death is fundamentally a bedside evaluation documenting the absence of clinical brainstem function, the inability to complete all clinical criteria mandates the use of an ancillary test. Young et al. ${ }^{18}$ review the appropriate use of ancillary testing, abandoning the electroencephalogram in favour of tests that demonstrate the absence of intracranial blood flow.

Based on an extensive review of death in acute care hospitals in Quebec, Cloutier et al. ${ }^{19}$ suggest that once brain death occurs, the diagnosis is not made in $24 \%$ of cases. In addition, once brain death is recognized and diagnosed, $23 \%$ of families are not approached for consent to donation. While these fundamental deficiencies in the process of donor identification and approach for consent may reflect national practice patterns, Baker et al. ${ }^{20}$ suggest that variations in donation are principally related to regional differences in demographics of brain injury, referral patterns and consent rates.

International comparative organ donor rates are routinely expressed 'per million population' and Barneih et al..$^{21}$ present the limitations of this benchmark comparison. Without correcting for regional differences in cerebrovascular and motor vehicle fatality rates, comparative statistics using the denominator of 'donors per million population' are inherently flawed. Given that the incidence of brain death is unknown, it is important to collect vital statistics that accurately reflect organ donation performance.

The final recommendations from the CCDT report on severe brain injury to neurological determination of death ${ }^{2}$ are the results of a national consensus collaborative between the disciplines involved in the care of these patients i.e., emergency medicine, trauma, neurosurgery, neurology, anesthesia and critical care. These recommendations were developed to address the aforementioned deficiencies, have been widely endorsed and are in the process of implementation.

The second forum sponsored by the CCDT was "Medical Management to Optimize Donor Organ Potential", held February 23-25 2004 in Mont Tremblant, in collaboration with the Canadian Critical Care Society, Canadian Society of Transplantation and the Canadian Association of Transplantation. Discussions were restricted to the interval of care that begins with the neurological determination of death and consent to organ donation and culminates with surgical organ procurement. During this period, there is significant opportunity for enhancing multi-organ function and improving organ utilization for the purposes of transplantation. This Forum was the first structured, cooperative assembly of health professionals in the critical care and transplantation fields and can be viewed as a landmark event in Canada.

Kutsogiannis et al. ${ }^{22}$ present an extensive literature review on organ donor management, highlighting the challenging pathophysiology, management strategies and the limitations to current levels of evidence in this developing field. Experimental and human studies have documented the profound cardiovascular dysfunction associated with brain death and support the use of hormonal therapy. ${ }^{23,24}$ The need for aggressive support of the myocardium and resuscitation of the cardiopulmonary system are emphasized, ${ }^{3}$ as organ donors have a high incidence of neurogenic myocardial dysfunction that is reversible with time and aggressive support in a substantial proportion. ${ }^{25}$ 
Aggressive hemodynamic support and the use of combined hormonal therapy (thyroid hormone, vasopressin, glucocorticoids) in donor management improves donor organ function, organ utilization and transplant outcomes. ${ }^{26,27}$

Badovinac et al..$^{28}$ present the demographic trends in Canadian organ donors, as characterized by an increasing age distribution and a shift toward cerebrovascular diseases as primary causes of death. Utilization rates for hearts and lungs have the greatest capacity for improvement. In order to understand factors influencing non-utilization, Hornby et al..$^{29}$ performed a four-centre Canadian review demonstrating variable practices around consent for individual organs and offering of organs consented. Suggestions for alternate management and transplant decision making, which could have resulted in organ utilization, were made in a significant proportion of heart and lung donors.

The donor management recommendations ${ }^{3}$ focus on reversible organ dysfunction and taking the time necessary in the ICU for aggressive cardiopulmonary resuscitation and vigilant monitoring to evaluate temporal changes in multi-organ function. All organs require consideration, as there are no predefined demographic or organ dysfunction thresholds that preclude consent to individual organs or offering of organs for transplantation.

In conclusion, end-of-life care in the ICU includes all efforts to actualize the desire and opportunity to donate organs. This evolving collaboration to establish best donor management practices in the ED, ICU and operating room must be linked to accurate performance markers and reciprocal collaboration by transplant specialties to ensure optimal organ utilization. In turn, improving donor identification, management and the utilization of organs must be linked to transplant graft and patient outcomes.

\section{Acknowledgements}

We express our sincere gratitude to our sponsors, the Canadian Council for Donation and Transplantation, and our utmost appreciation to the investigators, societies, organizations and expert participants who contributed to the success of these collective efforts and the possibility of the ensuing publications.

\section{De l'arrêt cérébral à la détermination neurologique de la mort et à l'utilisation d'organes : l'évolution du don d'organes en milieu hospitalier au Canada}

La planification du don d'organes a évolué grâce aux efforts faits par les services de transplantation pour répondre aux besoins des receveurs potentiels. Même si, au Canada, le taux de dons d'organes prélevés sur des cadavres témoigne d'un certain succès, il ne s'est pas amélioré. L'écart grandissant entre les besoins d'organes et leur disponibilité est bien connu et il a motivé l'action des gouvernements fédéral et provinciaux.

En septembre 1999, les ministres de la Santé, fédéral, provinciaux et territoriaux (FPT), ont accepté de formuler une stratégie FPT coordonnée pour améliorer le don d'organes et de tissus et le système de transplantation au Canada. En avril 2001, une composante clé de cette stratégie a été l'annonce de l'établissement du Conseil canadien pour le don et la transplantation (CCDT; www.ccdt.ca). Le mandat du Conseil est de donner des avis à la Conférence FPT des sous-ministres de la santé pour soutenir leurs efforts de coordination des activités FPT reliées au don et à la greffe d'organes et de tissus.

Le donneur d'organes en état de mort cérébrale est la principale source d'organes transplantables au Canada. Paradoxalement, les soins prodigués à ces individus sont du domaine exclusif de l'unité des soins intensifs (USI). Les spécialistes de la transplantation n'ont donc aucun rôle dans les soins aux donneurs éventuels, d'où leur capacité limitée à influencer ce domaine de pratique. Le Comité de don du CCDT a jugé nécessaire d'engager les soignants des USI à faire preuve de leadership en fixant des normes de pratique optimale dans ce domaine. Simultanément, la Société canadienne de soins intensifs (SCSI) a créé un groupe de travail sur le don d'organes et de tissus, ${ }^{1}$ qui a fortement appuyé les initiatives de collaboration en vue 
d'élaborer, d'appliquer et d'évaluer les processus pour accroître le don d'organes et de tissus à l'intérieur d'un cadre légal et éthique solide. La SCSI a insisté sur le fait que la possibilité de faire un don d'organes et de tissus soit présentée à toutes les familles admissibles et soit considérée comme une composante régulière des soins aux mourants à l'USI.

Souvent mal comprise, l'idée de mort cérébrale est mieux saisie par le terme d'arrêt cérébral. La situation est analogue à celle de l'arrêt cardiaque qui, une fois irréversible, amène la détermination de la mort de la personne par des critères cardiocirculatoires fondés sur l'absence de rythme cardiaque et de circulation. Or, après l'arrêt cérébral irréversible, la mort est déterminée par des critères neurologiques fondés sur l'absence de fonction du tronc cérébral. Cet acte est le processus et la procédure pour déterminer la mort.

Dans les récents numéros du Journal canadien d'anesthésie, dont le présent numéro, une série d'articles appuient les nouvelles recommandations unanimes sur la détermination neurologique de la $\operatorname{mort}^{2}$ et la prise en charge du donneur d'organes. ${ }^{3}$ Ces articles résultent d'une collaboration nationale à l'amélioration du processus et des procédures permettant de répondre au vœu du donneur et impliquent une pratique différente dans les services d'urgence, des neurosciences, des soins intensifs, de l'anesthésie, de la chirurgie et de la salle d'opération.

L'accès au don d'organes commence par une forme sévère et dévastatrice de lésion cérébrale qui dégénère en arrêt cérébral. Si l'arrêt du cerveau est complet et irréversible, les procédures de diagnostic doivent être entreprises pour confirmer la détermination neurologique de la mort. Il faut offrir aux familles la possibilité d'un don et si le consentement est acquis, le processus aboutit au prélèvement chirurgical des organes de donneurs consentants. Lors de la première réunion du CCDT, «De la lésion cérébrale sévère à la détermination neurologique de la mort», tenue du 9 au 11 avril 2003 à Vancouver, le mécanisme et les traitements qui influencent cette période de soins ont été discutés. ${ }^{4}$

Un patient dont le cerveau est sérieusement atteint doit être transporté au service des urgences (SU) et transféré ensuite à l'USI pour des soins neuroprotecteurs ou des soins aux mourants. Les caractéristiques de l'identification d'un donneur au SU et du triage entre le SU et l'USI dans des centres neurochirurgicaux de l'Ontario (CNO) sont revues par Tenn-Lyn et coll. ${ }^{5}$ Un nombre significatif de patients transférés aux CNO présentent une lésion qui semble progresser vers la mort cérébrale, mais peu de patients dans cette situation sont reconnus comme tels. Rocker et coll. ${ }^{6}$ discutent de la variabilité des soins aux mourants à l'USI, soins qui peuvent avoir des conséquences pour les patients atteints de lésion cérébrale sévère. Les traitements neuroprotecteurs évoluent, peuvent améliorer les suites d'une lésion cérébrale sévère ${ }^{7-9} \mathrm{et}$, donc, remettre en cause la façon de traiter et de prendre des décisions de fin de vie au SU et à l'USI.

Les perspectives historiques et l'évolution des critères de mort cérébrale sont fournies par Baron et coll. ${ }^{10}$ Quand un arrêt cérébral complet et irréversible survient, il doit être reconnu et diagnostiqué de façon fiable avec clarté et régularité. Le concept a beau être solide, les pratiques sont très variables. Les lignes directrices élaborées par le Comité du congrès canadien sur la mort cérébrale ${ }^{11}$ en 1988 et par le Groupe canadien sur les soins neurocritiques ${ }^{12}$ en 1999 , ont porté sur la clarification des critères, mais n'ont pas mené à une pratique uniforme. Hornby et coll. ${ }^{13}$ ont passé en revue les lignes directrices sur la mort cérébrale en milieu hospitalier canadien pour adultes et enfants et ont confirmé le manque d'homogénéité important et préoccupant des pratiques ${ }^{14,15}$ et de la documentation 16 décrites dans d'autres pays. Ces observations confirment la nécessité de normes nationales pour éliminer la variabilité diagnostique et améliorer la documentation, ce qui a été corroboré par une enquête auprès des médecins de soins intensifs canadiens menée par Doig et coll. ${ }^{17}$ Alors que la détermination neurologique de la mort est essentiellement une évaluation faite au chevet du malade qui documente l'absence de fonction clinique du tronc cérébral, l'incapacité à répondre à tous les critères cliniques exige le recours à un test auxiliaire. Young et coll. ${ }^{18}$ revoient la pertinence de tests auxiliaires, abandonnant l'électroencéphalogramme pour des tests démontrant l'absence de débit sanguin intracranien.

En s'appuyant sur une vaste revue sur la mort dans les hôpitaux de soins actifs du Québec, Cloutier et coll. ${ }^{19}$ montrent l'absence de diagnostic dans $24 \%$ des cas de mort cérébrale. De plus, une fois la mort cérébrale reconnue et diagnostiquée, $23 \%$ des familles ne sont pas informées de la possibilité d'un don d'organes. Ces déficiences fondamentales dans l'identification de donneurs et la consultation des familles, pour obtenir un consentement, pourraient représenter la pratique nationale, mais Baker et coll. ${ }^{20}$ pensent que les variations sont surtout reliées à des différences régionales de caractéristiques démographiques des patients atteints de lésion cérébrale, aux différentes façons de diriger les patients vers les services appropriés et à des taux de consentement différents.

Le taux international de donneurs d'organes est habituellement exprimé «par million de population». 
Barneih et coll. ${ }^{21}$ présentent les limites de ce critère de comparaison. Sans correction pour les différences régionales des taux de décès par accident vasculaire cérébral et accident d'automobile, les statistiques comparatives utilisant le dénominateur «donneurs par million de population» sont inexactes au départ. L'incidence de mort cérébrale étant inconnue, il faut recueillir des statistiques démographiques exactes sur la performance des dons d'organes.

Les recommandations finales qui ressortent du rapport du CCDT sur les lésions cérébrales sévères menant à la détermination neurologique de la mort $^{2}$ sont le résultat d'un consensus national de collaboration entre les disciplines concernées par les soins de ces patients : la médecine d'urgence, la traumatologie, la neurochirurgie, la neurologie, l'anesthésie et les soins intensifs. Ces recommandations voulaient régler les déficiences susmentionnées. Elles ont été largement appuyées et leur mise en œuvre est active.

Le second forum organisé par le CCDT, «Gestion des services médicaux pour maximiser le potentiel de compatibilité de l'organe du donneur», a eu lieu du 23 au 25 février 2004 à Mont Tremblant, en collaboration avec la Société canadienne de soins intensifs, la Société canadienne de transplantation et l'Association canadienne de transplantation. Les discussions ont porté sur l'intervalle de soins qui débute avec la détermination neurologique de la mort et le consentement au don d'organes et se termine avec le prélèvement chirurgical des organes. Pendant ce laps de temps, il est fort possible d'améliorer la fonction et le mode d'utilisation de nombreux organes. Ce forum a été la première collaboration structurée de professionnels en soins intensifs et en transplantation et peut être considérée comme un événement charnière au Canada.

Kutsogiannis et coll. ${ }^{22}$ présentent une vaste revue sur le traitement des donneurs d'organes, illustrant le défi physiopathologique, les stratégies thérapeutiques et les limites liées aux connaissances actuelles dans ce domaine. Les études en laboratoire et chez les humains ont montré une dysfonction cardiovasculaire très importante liée à la mort cérébrale et elles appuient l'usage d'une hormonothérapie. ${ }^{23,24}$ La nécessité d'un maintien dynamique du myocarde et de la réanimation du système cardiopulmonaire y est soulignée, ${ }^{3}$ car il y a chez les donneurs d'organes une forte incidence de dysfonction myocardique neurogène, réversible dans une large proportion avec le temps et un soutien constant. ${ }^{25}$ L'assistance hémodynamique active et l'usage combiné d'hormonothérapie (hormone thyroïdienne, vasopressine, glucocorticoïdes) améliorent la fonction des organes du donneur, leur utilisation et les suites de la transplantation. ${ }^{26,27}$
Badovinac et coll. ${ }^{28}$ présentent l'évolution démographique des donneurs d'organes canadiens dont l'âge est croissant et dont un plus grand nombre meurent de maladies cérébrovasculaires. Les taux d'utilisation du cour et des poumons sont les plus susceptibles de s'améliorer. Pour comprendre les facteurs qui influencent la non-utilisation, Hornby et coll. $^{29}$ ont réalisé une revue canadienne de quatre centres qui démontre des pratiques variables relatives au consentement au don d'organes et à l'offre d'organes consentie. Des suggestions sont faites pour modifier les traitements et la prise de décisions qui pourraient permettre l'utilisation d'organes chez une grande partie des donneurs de cœur et de poumons.

Les recommandations sur le traitement des donneurs ${ }^{3}$ insistent sur le caractère réversible de la dysfonction organique et le fait de prendre le temps nécessaire à l'USI pour la réanimation cardiopulmonaire agressive et le monitorage vigilant permettant d'évaluer les changements temporels de la fonction de nombreux organes. Il faut tenir compte de tous les organes, puisqu'il n'y a pas de données démographiques ou de dysfonction organique prédéfinies qui limitent le consentement au don d'organes spécifiques ou à l'offre d'organes pour la transplantation.

Enfin, les soins de fin de vie à l'USI incluent tous les efforts visant à actualiser le souhait et la possibilité de donner des organes. La collaboration grandissante pour établir les meilleures pratiques de soins aux donneurs dans les SU, les USI et les salles d'opération doit être en lien avec des marqueurs de performance précis et une collaboration réciproque de spécialistes de la transplantation pour s'assurer d'une utilisation optimale des organes. L'amélioration de l'identification des donneurs, du traitement et de l'utilisation des organes devront être successivement liés à un greffon transplanté et à des résultats pour le patient.

\section{Remerciements}

Nous tenons à remercier sincèrement notre commanditaire, le Conseil canadien pour le don et la transplantation et à exprimer une grande reconnaissance aux chercheurs, sociétés, organisations et experts participants qui ont contribué au succès des efforts collectifs et à la possibilité subséquente de publier.

\section{References}

1 Rocker GM. Organ and tissue donation in the intensive care unit. CMAJ 2002; 167: 1248-9.

2 Shemie SD, Doig C, Dickens B, et al.; Severe brain injury to neurological determination of death: Canadian forum recommendations. CMAJ 2006; 174: S1-13.

3 Shemie SD, Ross H, Pagliarello J, et al. Organ donor 
management in Canada: recommendations of the forum on Medical Management to Optimize Donor Organ Potential. CMAJ 2006; 174: S13-32.

4 Shemie SD, Doig C, Belitsky P. Advancing toward a modern death: the path from severe brain injury to neurological determination of death. CMAJ 2003; 168: 993-5.

5 Tenn-Lyn NA, Doig CJ, Shemie SD, Teitelbaum J, Cass $D E$. Potential organ donors referred to Ontario neurosurgical centres. Can J Anesth 2006; 53: 732-7.

6 Rocker GM, Cook DJ, Shemie SD. Brief review: Practice variation in end of life care in the ICU: implications for patients with severe brain injury. Can J Anesth 2006; 53: 814-9.

7 Bernard SA, Gray TW, Buist MD, et al. Treatment of comatose survivors of out-of-hospital cardiac arrest with induced hypothermia. N Engl J Med 2002; 346: 557-63

8 Schwab S, Steiner T, Aschoff A, et al. Early hemicraniectomy in patients with complete middle cerebral artery infarction. Stroke 1998; 29: 1888-93.

9 Trooskin SZ, Copes WS, Bain LW, Peitzman AB, Cooney $R N$, Jubeliver $R A$. Variability in trauma center outcomes for patients with moderate intracranial injury. J Trauma 2004; 57: 998-1005.

10 Baron L, Shemie SD, Teitelbaum J, Doig CJ. Brief review: History, concept and controversies in the neurological determination of death. Can J Anesth 2006; 53: 602-8.

11 Anonymous. Death and brain death: a new formulation for Canadian medicine. Canadian Congress Committee on Brain Death. CMAJ 1988; 138: 405-6.

12 Anonymous. Guidelines for the diagnosis of brain death. Canadian Neurocritical Care Group. Can J Neurol Sci 1999; 26: 64-6.

13 Hornby K, Shemie SD, Teitelbaum J, Doig C. Variability in hospital-based brain death guidelines in Canada. Can J Anesth 2006; 53: 613-9.

14 Wijdicks EF. Brain death worldwide: accepted fact but no global consensus in diagnostic criteria. Neurology 2002; 58: 20-5.

15 Powner DJ, Hernandez M, Rives TE. Variability among hospital policies for determining brain death in adults. Crit Care Med 2004; 32: 1284-8.

16 Wang MY, Wallace P, Gruen JP. Brain death documentation: analysis and issues. Neurosurgery 2002; 51 : 731-5.

17 Doig CJ, Young K, Teitelbaum J, Shemie SD. Brief survey: Determining brain death in Canadian intensive care units. Can J Anesth 2006; 53: 609-12.

18 Young GB, Shemie SD, Doig CJ, Teitelbaum J. Brief review: The role of ancillary tests in the neurological determination of death. Can J Anesth 2006; 53: 620-7.
19 Cloutier R, Baran D, Morin JE, et al. Brain death diagnoses and evaluation of the number of potential organ donors in Québec hospitals. Can J Anesth 2006; 53 : 716-21.

20 Baker A, Beed S, Fenwick J, et al. Number of deaths by neurological criteria, and organ and tissue donation rates at three critical care centres in Canada. Can J Anesth 2006; 53: 722-6.

21 Barnieh L, Baxter D, Boiteau P, Manns B, Doig C. Benchmarking performance in organ donation programs: dependence on demographics and mortality rates. Can J Anesth 2006; 53: 727-31.

22 Kutsogiannis DJ, Pagliarello G, Doig C, Ross H, Shemie $S D$. Medical management to optimize donor organ potential: review of the literature. Can J Anesth 2006; 53: 820-30.

23 Novitzky D. Detrimental effects of brain death on the potential organ donor. Transplant Proc 1997; 29: 3770-2.

24 Novitzky D, Wicomb WN, Cooper DK, Rose AG, Fraser $R C$, Barnard CW. Electrocardiographic, hemodynamic and endocrine changes occurring during experimental brain death in the chacma baboon. Heart Transplantation 1984; 4: 63-9.

25 Babcock WD, Menza RL, Zaroff JG. Increased donor heart utilization using serial echocardiography during donor management. J Heart Lung Transplant 2003; 22(1S): 74 .

26 Rosendale JD, Kauffman HM, McBride MA, et al. Hormonal resuscitation yields more transplanted hearts, with improved early function. Transplantation 2003; 75: 1336-41.

27 Rosendale JD, Kauffman HM, McBride MA, et al. Hormonal resuscitation associated with more transplanted organs with no sacrifice in survival. Transplantation 2004; 78(Sup 2): 17.

28 Badovinac K, Greig PD, Ross H, Doig CJ, Shemie SD. Organ utilization among deceased donors in Canada, 1993-2002. Can J Anesth 2006; 53: 838-44.

29 Hornby K, Ross H, Keshavjee S, Rao V, Shemie SD. Non-utilization of hearts and lungs after consent for donation: a Canadian multicentre study. Can J Anesth 2006; 53: 831-7. 